\title{
О ПРОНОРМАЛЬНОСТИ ВТОРЫХ МАКСИМАЛЬНЫХ ПОДГРУПП В КОНЕЧНЫХ ГРУППАХ С ЦОКОЛЕМ $L_{2}(q)^{1}$
}

\section{В. И. Зенков}

\begin{abstract}
Согласно $\Phi$. Холлу подгруппа $H$ конечной группы $G$ называется пронормальной в $G$, если для любого элемента $g$ из $G$ подгруппы $H$ и $H^{g}$ сопряжены в $\left\langle H, H^{g}\right\rangle$. Простейшими примерами пронормальных подгрупп конечных групп служат нормальные подгруппы, максимальные подгруппы, силовские подгруппы. Пронормальные подгруппы конечных групп исследовались рядом авторов. Так, Леговини (1981) изучала конечные группы, в которых каждая подгруппа субнормальна или пронормальна. Позднее Ли и Чжан (2013) описали строение конечной группы $G$, в которой для второй максимальной подгруппы $H$ ее индекс в $\left\langle H, H^{g}\right\rangle$ не содержит квадратов для любого элемента $g$ из $G$. В целом ряде работ Е. П. Вдовина, А. С. Кондратьева, Н. В. Масловой и Д. О. Ревина (2012-2019) рассматривались пронормальности подгрупп в конечной простой неабелевой группе и, в частности, существование в конечной простой неабелевой группе непронормальной подгруппы нечетного индекса. Автор поставил вопрос 19.109 в "Коуровской тетради" о равносильности в конечной простой неабелевой группе условия пронормальности ее вторых максимальных подгрупп и условия холловости ее максимальных подгрупп. В. Н. Тютяновым был указан контрпример $L_{2}\left(2^{11}\right)$ к зтому вопросу. В данной работе даются необходимые и достаточные условия пронормальности вторых максимальных подгрупп в группе $L_{2}(q)$. Кроме того, при $q \leq 11$ определены конечные почти простые группы с цоколем $L_{2}(q)$, в которых все вторые максимальные подгруппы пронормальны.
\end{abstract}

Ключевые слова: конечная группа, простая группа, максимальная подгруппа, пронормальная подгруппа.

V. I. Zenkov. On pronormality of second maximal subgroups in finite groups with socle $L_{2}(q)$.

According to $\mathrm{Ph}$. Hall, a subgroup $H$ of a finite group $G$ is called pronormal in $G$ if, for any element $g$ of $G$, the subgroups $H$ and $H^{g}$ are conjugate in $\left\langle H, H^{g}\right\rangle$. The simplest examples of pronormal subgroups of finite groups are normal subgroups, maximal subgroups, and Sylow subgroups. Pronormal subgroups of finite groups were studied by a number of authors. For example, Legovini (1981) studied finite groups in which every subgroup is subnormal or pronormal. Later, Li and Zhang (2013) described the structure of a finite group $G$ in which, for a second maximal subgroup $H$, its index in $\left\langle H, H^{g}\right\rangle$ does not contain squares for any $g$ from $G$. A number of papers by Kondrat'ev, Maslova, Revin, and Vdovin (2012-2019) are devoted to studying the pronormality of subgroups in a finite simple nonabelian group and, in particular, the existence of a nonpronormal subgroup of odd index in a finite simple nonabelian group. In the Kourovka Notebook, the author formulated Question 19.109 on the equivalence in a finite simple nonabelian group of the condition of pronormality of its second maximal subgroups and the condition of Hallness of its maximal subgroups. Tyutyanov gave a counterexample $L_{2}\left(2^{11}\right)$ to this question. In the present paper, we provide necessary and sufficient conditions for the pronormality of the second maximal subgroups in the group $L_{2}(q)$. In addition, for $q \leq 11$, we find the finite almost simple groups with socle $L_{2}(q)$ in which all second maximal subgroups are pronormal.

Keywords: finite group, simple group, maximal subgroup, pronormal subgroup.

MSC: 20D06, 20D30, 20E28

DOI: $10.21538 / 0134-4889-2020-26-3-32-43$

\section{Введение}

Согласно $\Phi$. Холлу подгруппа $H$ конечной группы $G$ называется пронормальной в $G$, если для любого элемента $g$ из $G$ подгруппы $H$ и $H^{g}$ сопряжены в $\left\langle H, H^{g}\right\rangle$. Простейшими примерами пронормальных подгрупп конечных групп служат нормальные подгруппы, максимальные подгруппы, силовские подгруппы, холловы подгруппы разрешимых групп. Пронормальные

\footnotetext{
${ }^{1}$ Работа выполнена при финансовой поддержке проекта повышения конкурентоспособности ведущих университетов России (соглашение 02.А03.210006 от 27.08.2013).
} 
подгруппы конечных групп исследовались рядом авторов. Так, Леговини [1] изучала конечные группы, в которых каждая подгруппа субнормальна или пронормальна. Позднее Ли и Чжан [2] описали строение конечной группы $G$, в которой для второй максимальной подгруппы $H$ ее индекс в $\left\langle H, H^{g}\right\rangle$ не содержит квадратов для любого элемента $g$ из $G$. В работе Е. П. Вдовина и Д. О. Ревина [3] было доказано, что в любой конечной простой группе любая ее холлова подгруппа пронормальна. В этой же работе была сформулирована гипотеза о пронормальности подгрупп нечетного индекса в конечных простых группах. А. С. Кондратьев, Н. В. Маслова и Д. О. Ревин подтвердили эту гипотезу для многих классов простых групп в [4] и опровергли ее в общем случае в [5]. В последующих работах рассматривался вопрос о существовании в конечной простой группе непронормальной подгруппы нечетного индекса. В "Коуровской тетради" [6] автором был поставлен следующий вопрос 19.109: "Верно ли, что в конечной простой неабелевой группе $G$ следующие условия равносильны: (1) все максимальные в $G$ подгруппы являются холловыми, $(2)$ все вторые максимальные в $G$ подгруппы пронормальны?" Отрицательный ответ на этот вопрос дал В. Н. Тютянов, приведя пример группы $G=L_{2}\left(2^{11}\right)$, в которой все вторые максимальные подгруппы пронормальны, однако диэдральные максимальные подгруппы порядков $2\left(2^{11} \pm 1\right)$ не являются в ней холловыми.

Указанные выше вопрос из "Коуровской тетради" и пример В.Н. Тютянова послужили отправной точкой для изучения в данной работе конечных групп с цоколем $L_{2}(q)$, в которых все вторые максимальные подгруппы пронормальны. Наш подход основан на классификации максимальных подгрупп в группе $L_{2}(q)$ (см. [7-10]).

Как оказалось, имеется существенное различие в решении этого вопроса в зависимости от q. В теоремах 1, 2 даны необходимые и достаточные условия для пронормальности всех вторых максимальных подгрупп в группе $L_{2}(q)$.

Теорема 1. Пусть $G=L_{2}(q)$, где $q=2^{n} \geq 4$. Тогда следующие условия равносильны:

(1) все вторые максимальные подгруппы группы $G$ пронормальны в $G$;

(2) число q-1 не является простым числом Мерсенна.

Теорема 2. Пусть $G=L_{2}(q)$, где q нечетно и $q>11$. Тогда следующие условия равносильны:

(1) все вторые максимальные подгруппы группы $G$ пронормальны в $G$;

(2) $q$ - простое число, числа $\frac{q-1}{2} u \frac{q+1}{2}$ не простые, $\left(q^{2}-1\right)_{2} \leq 2^{5}$ и если $q \equiv$ $\varepsilon(\bmod 8)$, где $\varepsilon= \pm 1$, mo $q \equiv-\varepsilon(\bmod 3) \quad u \quad q \equiv-\varepsilon(\bmod 15)$ npu $q \equiv \pm$ $1(\bmod 10)$.

Легко проверить, что при $q \in\{17,89\}$ условие (2) в теореме 2 выполняется.

Кроме того, при $q \leq 11$ определены конечные почти простые группы с цоколем $L_{2}(q)$, в которых все вторые максимальные подгруппы пронормальны. Доказана

Теорема 3. Пусть $G-$ конечнал группа с иоколем $L_{2}(q)$, где $q \leq 11$. Тогда следующие условия равносильны:

(1) в группе $G$ все вторые максимальные подгруппы пронормальны в $G$;

(2) $G \simeq \operatorname{Aut}\left(L_{2}(4)\right), L_{2}(7)$, Aut $\left(L_{2}(8)\right)$ или $L_{2}(11)$.

Как следует из теоремы 3, положения теоремы 2 нарушаются при $q \in\{7,11\}$, а теорема 1 справедлива и при $q \in\{4,8\}$.

\section{1. Обозначения и предварительные результаты}

В работе будем в основном придерживаться обозначений из [11] и терминологии из публикаций $[8 ; 12]$. Через $A 入 B$ или $A: B$ обозначается полупрямое произведение группы $A$ на 
группу $B$, а через $\operatorname{Soc}(G)$ - цоколь конечной группы $G$, т. е. подгруппа, порожденная всеми ее минимальными нормальными подгруппами. Запись $M<\cdot G$ означает, что $M$ - максимальная подгруппа в группе $G$. Если $H<\cdot M<\cdot G$, то $H$ называется второй максимальной подгруппой в $G$.

В работе часто будет использоваться

Предложение. Пусть $G$ - конечная группа и $H$ - вторая максимальная подгруппа группы $G$, не пронормальная в $G$. Тогда выполняются следующие утверждения:

(1) найдется элемент $g \in G$ такой, что

$$
F:=\left\langle H, H^{g}\right\rangle \neq G, \quad H^{f} \neq H^{g},
$$

для всех $f \in F, H<\cdot M<\cdot G$ и $H<F \leq M_{1}<\cdot G$ для некоторых максимальных подгрупп $M u$ $M_{1}$ из $G$;

(2) если $H$ является $p$-подгруппой для некоторого простого числа $p$ и $F_{1}$ - силовская p-подгруппа в $F$, содержащая $H$, то $H$ не слабо замкнута в $F_{1}$ относительно $G$;

(3) если $H<\cdot F$, то $H$ не содержст нормальных в $H$ подгрупп, которые суть силовские подгруппы в $F$, но не являются нормальными в $F$.

Д о к а з а т е л ь с т в о. Утверждение (1) вытекает из определения пронормальности подгруппы.

Покажем, что утверждения (2) и (3) следуют из (1).

Допустим, что $(2)$ неверно. Тогда подгруппа $H$ слабо замкнута в $F_{1}$ относительно $G$ и, таким образом, $F_{1}^{g}=F_{1}^{f}$ для некоторого элемента $f$ из $F$, откуда $H^{g}=H^{f}$, что противоречит п. (1).

Допустим, что (3) не верно. Тогда $H$ содержит нормальную подгруппу $H_{1}$, которая является силовской подгруппой в $F$ и не нормальна в $F$. Но тогда $H_{1}^{g}=H_{1}^{f}$ для некоторого элемента $f$ из $F$. Если при этом $H^{f} \neq H^{g}$, то подгруппа $H_{1}^{f}$ нормализуется подгруппами $H^{f}$ и $H^{g}$ и, следовательно, подгруппа $H_{1}^{f}$ нормальна в $F$. Но отсюда подгруппа $H_{1}$ нормальна в $F$, а это не так. Поэтому $H^{f}=H^{g}$, что противоречит п. (1).

Нам понадобятся также сведения о максимальных подгруппах в группах $L_{2}(q)$ и $P G L_{2}(q)$ (см. [7-10]).

Лемма 1 [9, теорема 2.1]. Пусть $q=2^{f} \geq 4$, где $f$ - натуральное число. Тогда максимальные подгруппы в $L_{2}(q)$ изоморфны одной из следующих групп:
(a) $C_{2}^{f} \lambda C_{q-1}$;
(б) $D_{2(q-1)}$;
(в) $D_{2(q+1)}$;
(г) $L_{2}\left(q_{0}\right)$, где $q=q_{0}^{r}$ для некоторого простого числа $r$ и $q_{0} \neq 2$.

Лемма 2 [9, теорема 2.2]. Пусть $q=p^{f} \geq 5$, где $p$ - нечетное простое число и $f$ натуральное число. Тогда максимальные подгруппы из $L_{2}(q)$ изоморфны одной из следующих гpynn:
(a) $C_{p}^{f} \lambda C_{\frac{(q-1)}{2}}$;
(б) $D_{q-1} \partial л я q \geq 13$;
(в) $D_{q+1}$ для $q \neq 7,9$;
(г) $P G L_{2}\left(q_{0}\right) \partial л я q=q_{0}^{2}($ два сопрлженных класса);
(д) $P S L_{2}\left(q_{0}\right)$ при $q=q_{0}^{r}$ для нечетного простого числа $r$;
(з) $A_{4}$ для $q=p \equiv \pm 3(\bmod 8) u q \not \equiv \pm 1(\bmod 10)$;
(и) $S_{4}$ для $q=p= \pm 1(\bmod 8)($ два сопряљенных класса).

(ж) $A_{5}$ для $q \equiv \pm 1(\bmod 10)$, где или $q=p$, или $q=p^{2} u p \equiv \pm 3(\bmod 10)$ (два сопряљсенных класса); 
Лемма 3 [9, теорема 3.5]. Пусть $q=p^{f}>3$, где $p$ - нечетное простое число u $f$ - натуральное число. Тогда максимальные подгруппы в $P G L_{2}(q)$, не содержащие $L_{2}(q)$, изоморфны одной из следующих групп:
(a) $C_{p}^{f} \lambda C_{q-1}$;
(б) $D_{2(q-1)}$ для $q \neq 5$;
(в) $D_{2(q+1)}$;
(г) $S_{4}$ для $q=p \equiv \pm 3(\bmod 8)$;
(д) $P G L\left(2, q_{0}\right)$ при $q=q_{0}^{r}$ для нечетного простого числа $r$.

\section{2. Доказательство теоремы 3}

Лемма 4. Пусть $G-$ конечная группа с $\operatorname{Soc}(G) \simeq L_{2}(4) \simeq L_{2}(5) \simeq A_{5}$. Тогда следующие условия равносильны:

(1) все вторые максимальные подгруппы группы $G$ пронормальны в $G$;

(2) $G \simeq \operatorname{Aut}\left(L_{2}(4)\right) \simeq S_{5}$.

Д о к а з а т е л ь с т в о. Докажем, что $(1) \Rightarrow(2)$. Действительно, по [7, с. 2] либо $G \simeq$ $L_{2}(4)$, либо $G \simeq S_{5}$. Предположим, что $G \simeq L_{2}(4)$. Тогда, согласно [11, с. 2] $G$ содержит максимальные подгруппы, изоморфные как $D_{10}$, так и $D_{6}$. Следовательно, подгруппа $T$ второго порядка из $G$ является второй максимальной подгруппой в $G$. Однако $T$ содержится в четверной силовской 2-подгруппе $S$ группы $G$ и $N_{G}(S) \simeq A_{4}$ действует транзитивно на инволюциях из $N_{G}(S)$. Поэтому $T \neq T^{f}$ для некоторого элемента $f$ из $N_{G}(S)$. Но $\left[T, T^{f}\right]=1$. Отсюда $\left\langle T, T^{f}\right\rangle=T \times T^{f}$ и, таким образом, подгруппа $T$ не пронормальна в $G$. Следовательно, $(1) \Rightarrow(2)$.

Докажем, что $(2) \Rightarrow(1)$. Предположим, что это неверно. Тогда группа $G \simeq S_{5} \simeq P G L_{2}(5)$ содержит непронормальную вторую максимальную подгруппу $H$. Применим предложение в его обозначениях. Имеем $H<\cdot M<\cdot G$ и $H<F \leq M_{1}<\cdot G$ для некоторых максимальных подгрупп $M$ и $M_{1}$ из $G$. По лемме 3 каждая из подгрупп $M$ и $M_{1}$ изоморфна $2 \times D_{6}, 5: 4, S_{4}$ или $A_{5}$. Рассмотрим все эти случаи.

(a) $M \simeq 2 \times D_{6}$. Тогда $H$ изоморфна $2^{2}, D_{6}$ или $C_{6}$.

(a1) $H \simeq 2^{2}$. Тогда $H \not \subset G^{\prime} \simeq A_{5}$, поэтому либо $F=M$, что невозможно по утверждению (2) предложения, либо $F=M_{1}$, где $M_{1} \simeq S_{4}$, что также невозможно по утверждению (2) предложения ввиду слабой замкнутости относительно $G$ четверной подгруппы $H$ в силовской 2-подгруппе из $F$, содержащей $H$.

(а2) $H \simeq D_{6}$. Имеем либо $H \not \subset G^{\prime} \simeq A_{5}$ и тогда $F \leq M$ или $M_{1} \simeq S_{4} \simeq F$, либо $F \leq G^{\prime} \simeq A_{5}$ и $F \simeq A_{5}$. Случаи $M_{1} \simeq S_{4}, A_{5}$ невозможны по утверждению (3) предложения. Значит, $F \leq M$. Но $H<\cdot M$, таким образом, $F=M$. Поэтому подгруппа $O(H)=O(F)=O(M)$ характеристична в $F$ и, следовательно, $O(H)=O(H)^{g}$. Но тогда $g \in N_{G}(O(H))=M=F$, что противоречит утверждению (1) предложения.

(а3) $H \simeq C_{6}$. Тогда $H \not \subset G^{\prime} \simeq A_{5}$ и отсюда $F \leq M$. Теперь получаем противоречие, как в случае (а2).

(б) $M \simeq 5: 4$. Имеем либо $H \not \leq G^{\prime} \simeq A_{5}$ и тогда $F=M$ и $H \simeq C_{4}$, либо $F \leq G^{\prime} \simeq$ $A_{5}$ и $F \simeq A_{5}$ и $H \simeq 5: 2$. Первый случай невозможен по утверждению (2) предложения ввиду слабой замкнутости подгруппы $H$ в силовской 2-подгруппе из $F$, содержащей $H$. Второй случай невозможен по утверждению (3) предложения.

(в) $M \simeq S_{4}$. Имеем либо $H \not \subset G^{\prime} \simeq A_{5}$ и тогда либо $F=M$ и $H \simeq D_{8}$, либо $F=M_{1} \simeq 2 \times D_{6}$ и $H \simeq D_{6}$, либо $H \leq G^{\prime} \simeq A_{5}, F \simeq A_{5}$ и $H \simeq A_{4}, D_{6}$. Случаи, в которых $H \simeq D_{6}$, разобраны в п. (а). Случай $H \simeq D_{8}$ невозможен по утверждению (2) предложения, а случай $H \simeq A_{4}$ невозможен по утверждению (3) предложения.

(г) $M \simeq A_{5}$. Здесь все возможности для $H$ и $F$ разобраны в предыдущих случаях (а,б,в). 
Лемма 5. Если $G-$ конечная группа $c$ Soc $(G) \simeq L_{2}(9) \simeq A_{6}$, mo $G$ содержсит непронормальную вторую максимальную подгруппу.

Д о к а з а т е л ь с т в о. Пусть $G \simeq L_{2}(9) \simeq A_{6}$. Тогда по лемме $2 G$ имеет два класса максимальных подгрупп, изоморфных $S_{4}$. Пусть $S_{4} \simeq M<\cdot G$ и $D_{6} \simeq H<\cdot M$. Покажем, что подгруппа $H$ не пронормальна в $G$. Подгруппа $H$ содержит нормальную подгруппу $M_{2}$ порядка 3 и $M_{2}<\cdot S \in \operatorname{Syl}_{3}(G)$. Ввиду [11, с. 4,5$]$ имеем $C_{G}\left(M_{2}\right)=S \simeq 3^{2}$ и $N_{G}(S)$ - группа Фробениуса вида $3^{2}: 4$, следовательно, $H<N_{G}\left(M_{2}\right) \leq N_{G}(S)$ и $\left|N_{G}(S): N_{G}\left(M_{2}\right)\right|=2$. В частности, $N_{G}\left(M_{2}\right)<M_{G}(S)$. Поэтому $\left\langle H, H^{g}\right\rangle \neq H$ для элемента $g$ из $N_{G}(S) \backslash N_{G}\left(M_{2}\right)$. Отсюда следует, что $\left\langle H, H^{g}\right\rangle=N_{G}\left(M_{2}\right)$. Поэтому $H$ не пронормальна в $G$.

Пусть $G \simeq \operatorname{Aut}\left(A_{6}\right) \simeq \operatorname{Aut}\left(S p_{4}(2)\right)$. С учетом [11, с. 4] $G$ имеет максимальную подгруппу $M \simeq S_{6} \simeq S p_{4}(2), M \simeq S p_{4}(2)$ имеет параболические максимальные подгруппы $P_{1}$ и $P_{2}$, которые изоморфны $2 \times S_{4}$ и не сопряжены в $M$, а $G$ содержит элемент порядка 4 , который переставляет между собой подгруппы $P_{1}$ и $P_{2}$. Поэтому вторая максимальная в $G$ подгруппа $P_{1}$ не пронормальна в $G$.

Таким образом, согласно $\left[11\right.$, с. 4] $G$ является расширением группы $L_{2}(9) \simeq A_{6}$ с помощью подгруппы порядка 2.

Пусть $G \neq S_{6}$. Тогда исходя из $[11$, с. 4] или лемм 2 и 3 Soc $(G)$ имеет два класса максимальных подгрупп, изоморфных $A_{5}$ или $S_{4}$ соответственно, и некоторый 2-элемент из $G \backslash \operatorname{Soc}(G)$ переставляет между собой эти два класса. Поэтому представители данных классов не пронормальны в $G$.

Пусть $G=S_{6}$. Согласно [11, с. 4] $G$ содержит максимальную подгруппу $M \simeq 2 \times S_{4}$. Рассмотрим в $M$ максимальную подгруппу $H \simeq 2 \times D_{6}$ и покажем, что $H$ не пронормальна в $G$. Имеем

$$
O_{3}(H)<S \in \operatorname{Syl}_{3}(G), \quad S \simeq 3^{2}, \quad N_{G}(S) \simeq D_{6} \prec 2 \text { и } \quad N_{G}\left(O_{3}(H)\right) \simeq D_{6} \times D_{6} .
$$

Поэтому $N_{G}\left(O_{3}(H)\right)$ - подгруппа индекса 2 в $N_{G}(S)$ и, следовательно, $O_{3}(H) \neq O_{3}(H)^{g}$ для некоторого элемента $g$ из $N_{G}(S)$. Поскольку подгруппы $\left\langle O_{3}(H), O_{3}(H)^{f}\right\rangle$ и $H$ нормализуют $O_{3}(H)$, подгруппа $H$ не пронормальна в $G$.

Лемма 6. Пусть $\operatorname{Soc}(G) \simeq L_{2}(7)$. Тогда следующие условия равносильны:

(1) все вторые максимальные подгруппы в группе $G$ пронормальны в $G$;

(2) $G \simeq L_{2}(7)$.

Д о к а з а т е л ь с т в о. Докажем, что $(1) \Rightarrow(2)$. Действительно, согласно $[11$, с. 3] либо $G \simeq \operatorname{Aut}\left(L_{2}(7)\right)$, либо $G \simeq L_{2}(7)$.

Предположим, что $G \simeq$ Aut $\left(L_{2}(7)\right)$. В соответствии с [11, с. 3] $G$ имеет максимальную подгруппу $M=\operatorname{Soc}(G) \simeq L_{3}(2)$, а $M$ - параболические максимальные подгруппы $P_{1}$ и $P_{2}$, которые изоморфны $S_{4}$ и не сопряжены в $M ; G$ содержит инволюцию, которая переставляет между собой подгруппы $P_{1}$ и $P_{2}$. Поэтому вторая максимальная в $G$ подгруппа $P_{1}$ не пронормальна в $G$.

Докажем, что $(2) \Rightarrow(1)$. Предположим, что это неверно. Тогда группа $G \simeq L_{2}(7)$ содержит непронормальную вторую максимальную подгруппу $H$. Применим предложение в его обозначениях. Имеем $H<\cdot M<\cdot G$ и $H<F \leq M_{1}<\cdot G$ для некоторых максимальных подгрупп $M$ и $M_{1}$ из $G$. По лемме 2 подгруппа $M$ изоморфна $7: 3$ или $S_{4}$. Если $M \simeq 7: 3, H \in \operatorname{Syl}_{3}(G)$ или $H \in \operatorname{Syl}_{7}(G)$, что противоречит утверждению (2) предложения. Отсюда $M \simeq S_{4}$ и, следовательно, максимальная в $M$ подгруппа $H$ изоморфна $D_{8}, A_{4}$ или $D_{6}$.

Случаи $H \simeq D_{8}$ и $H \simeq D_{6}$ противоречат утверждениям (2) и (3) предложения соответственно. Поэтому $H \simeq A_{4}$ и, таким образом, $F \simeq S_{4}$. Поскольку $H-$ единственная подгруппа индекса 2 в $F$, из $H^{g}<F$ вытекает, что $H^{g}=H$. Но это противоречит утверждению (1) предложения.

Следовательно, $(2) \Rightarrow(1)$. 
Лемма 7. Пусть $\operatorname{Soc}(G) \simeq L_{2}(8)$. Тогда следующие условия равносильнъ:

(1) все вторые максимальные подгруппы в группе $G$ пронормальны в $G$;

$(2) G \simeq \operatorname{Aut}\left(L_{2}(8)\right)$.

Д о к а з а т е л ь с т в о. Докажем, что $(1) \Rightarrow(2)$. Действительно, согласно $[7$, с. 6] либо $G \simeq \operatorname{Aut}\left(L_{2}(8)\right)$, либо $G \simeq L_{2}(8)$.

Предположим, что $G \simeq L_{2}(8)$. Тогда с учетом [11, с. 6] $G$ содержит максимальную подгруппу, изоморфную $D_{14}$. Следовательно, подгруппа $T$ второго порядка из $G$ является второй максимальной подгруппой в $G$. Однако $T$ содержится в силовской 2-подгруппе $S$ группы $G$ и $N_{G}(S) \simeq 2^{3}: 7$ действует транзитивно на инволюциях из $N_{G}(S)$. Поэтому $T \neq T^{f}$ для некоторого элемента $f$ из $N_{G}(S)$. Но $\left[T, T^{f}\right]=1$. Отсюда $\left\langle T, T^{f}\right\rangle=T \times T^{f}$ и, таким образом, подгруппа $T$ не пронормальна в $G$. Следовательно, $(1) \Rightarrow(2)$.

Докажем, что $(2) \Rightarrow(1)$. Предположим, что это неверно. Тогда группа $G \simeq \operatorname{Aut}\left(L_{2}(8)\right)$ содержит непронормальную вторую максимальную подгруппу $H$. Применим предложение в его обозначениях. Имеем $H<\cdot M<\cdot G$ и $H<F \leq M_{1}<\cdot G$ для некоторых максимальных подгрупп $M$ и $M_{1}$ из $G$. По лемме 1 подгруппа $M$ изоморфна $2^{3}: 7: 3,9: 6$ или $7: 6$. Рассмотрим все эти случаи.

(a) $M \simeq 2^{3}: 7: 3$. Тогда $H$ изоморфна $2^{3}: 7,2^{3}: 3$ или $7: 3$. Во всех этих случаях $H$ нормализует соответствующую силовскую подгруппу из $\operatorname{Soc}(G)$. Но это противоречит утверждению (3) предложения.

(б) $M \simeq 9: 6$. Тогда либо $H \simeq 3 \times D_{6}$, либо $H \simeq D_{18}$, либо $|H|=3^{3}$. Случаи $H \simeq D_{18}$ и $|H|=3^{3}$ противоречат утверждениям (3) и (2) предложения соответственно. Таким образом, $H \simeq 3 \times D_{6}$ и, следовательно, $F=M=M_{1}$. Но

$$
O(H)=\Omega(O(M))=\Omega(O(F)) .
$$

Поэтому из $H^{g}<F$ вытекает, что $g$ лежит в $N_{G}(\Omega(O(F)))=M=F$. Но это противоречит утверждению (1) предложения.

(в) $M \simeq 7: 6$. Тогда подгруппа $H$ изоморфна $7: 2,7: 3$ или $C_{6}$. Первые два случая противоречат утверждению (3) предложения. Таким образом, $H \simeq C_{6}$ и, следовательно, $\mathrm{O}_{2}(H)<\operatorname{Soc}(G)$. По лемме 1 подгруппа $M_{1}$ изоморфна $2^{3}: 7: 3,9: 6$ или $7: 6$.

Если $M_{1} \simeq 7: 6$,то $M=F$ и подгруппа $H$ является холловой в $F$, что противоречит утверждению (1) предложения.

Пусть $M_{1} \simeq 9: 6$. Из сопряженности силовских 2-подгрупп в $F$ для инволюции $i$ из $H$ имеем $i^{g}=i^{f}$ для некоторого $f \in F$. Ввиду утверждения (1) предложения получаем $H^{g} \neq H^{f}$ и, значит, $O(H)^{g} \neq O(H)^{f}$. Отсюда

$$
C_{G}\left(i^{f}\right)>\left\langle O(H)^{g}, O(H)^{f}\right\rangle \leq \Omega\left(O\left(M_{1}\right)\right) \simeq C_{3} \times C_{3} .
$$

Поэтому $\left\langle O(H)^{g}, O(H)^{f}\right\rangle \simeq \Omega(O(M))$. Но инволюция $i^{f}$ инвертирует неединичный центр подгруппы $O(M)$, который содержится в $\Omega(O(M))$; противоречие.

Таким образом, $M_{1} \simeq 2^{3}: 7: 3$. Исходя из $\left[7\right.$, с. 6] имеем $C_{M_{1}}\left(O_{3}(H)\right)=H$. Так как $O_{3}(H) \in$ $\operatorname{Syl}_{3}(F)$, то $O_{3}(H)^{g}=O_{3}\left(H^{f}\right)$ для некоторого $f$ из $F$. Ввиду утверждения (1) предложения выводим $H^{g} \neq H^{f}$ и, следовательно, $O_{2}(H)^{g} \neq O_{2}(H)^{f}$. Поскольку

$$
\left.\left\langle O_{2}(H)^{f}, O_{2}\left(H^{g}\right)\right\rangle \leq O_{2}\left(M_{1}\right)\right) \simeq 2^{3},
$$

получаем $\left|C_{G}\left(O_{3}(H)\right)\right|_{2} \geq 4$. Это противоречит тому, что $\left|C_{G}\left(O_{3}(H)\right)\right|_{2}=2$ согласно [7, с. 6]. Следовательно, $(2) \Rightarrow(1)$.

Лемма 8. Пусть $\operatorname{Soc}(G) \simeq L_{2}(11)$. Тогда следующие условия равносильны:

(1) все вторые максимальные подгруппь в группе $G$ пронормальны в $G$;

(2) $G \simeq L_{2}(11)$. 
Д о к а з а т е л ь с т в о. Докажем, что $(1) \Rightarrow(2)$. Согласно [11, с. 7] либо $G \simeq L_{2}(11)$, либо $G \simeq \operatorname{Aut}\left(L_{2}(11)\right) \simeq P G L_{2}(11)$.

Предположим, что $G \simeq \operatorname{Aut}\left(L_{2}(11)\right)$. Исходя из [11, с. 7] $G$ имеет максимальную подгруппу $M=\operatorname{Soc}(G) \simeq L_{2}(11)$, а $M$ - параболические максимальные подгруппы $P_{1}$ и $P_{2}$, которые изоморфны $A_{5}$ и не сопряжены в $M ; G$ содержит инволюцию, которая переставляет между собой подгруппы $P_{1}$ и $P_{2}$. Поэтому вторая максимальная в $G$ подгруппа $P_{1}$ не пронормальна в $G$. Следовательно, $(1) \Rightarrow(2)$.

Докажем, что $(2) \Rightarrow(1)$. Предположим, что это неверно. Тогда группа $G \simeq L_{2}(11)$ содержит непронормальную вторую максимальную подгруппу $H$. Применим предложение в его обозначениях. Имеем $H<\cdot M<\cdot G$ и $H<F \leq M_{1}<\cdot G$ для некоторых максимальных подгрупп $M$ и $M_{1}$ из $G$. По лемме 2 каждая из подгрупп $M$ и $M_{1}$ изоморфна $11: 5, D_{12}$ или $A_{5}$. Рассмотрим все эти случаи.

(а) $M \simeq 11: 5$. Тогда $H$ является силовской подгруппой в $G$, что противоречит утверждению (2) предложения.

(б) $M \simeq D_{12}$. Тогда $H$ изоморфна $2^{2}, S_{3}$ или $C_{6}$.

Если $H \simeq 2^{2}$, то является силовской 2-подгруппой в $G$, что противоречит утверждению (2) предложения.

Если $H \simeq C_{6}$, то $M_{1} \simeq D_{12}$ и, следовательно, $O_{3}(H)=O_{3}(F)=O_{3}\left(M_{1}\right)$ и $F=M_{1}$. Поскольку $H^{g}<F$, имеем

$$
g \in N_{G}\left(O_{3}(H)\right)=F=M,
$$

что противоречит утверждению (1) предложения.

Таким образом, $H \simeq S_{3}$ и отсюда подгруппа $M_{1}$ изоморфна $D_{12}$ или $A_{5}$. Если $M_{1} \simeq D_{12}$, то, рассуждая как в предыдущем абзаце, получим противоречие с утверждением (1) предложения. Поэтому $M_{1} \simeq A_{5}$ и, значит, $F=M_{1}$, что противоречит утверждению (3) предложения.

(в) $M \simeq A_{5}$. Тогда $H$ изоморфна $5: 2, S_{3}$ или $A_{4}$. Если $H$ изоморфна $5: 2$ или $A_{4}$, то $F=M_{1} \simeq A_{5}$, что противоречит утверждению (3) предложения. Поэтому $H \simeq S_{3}$. Рассуждая, как в случае (б), приходим к противоречию.

Следовательно, $(2) \Rightarrow(1)$.

Теорема 3 следует из лемм 4-8.

\section{3. Доказательство теоремы 1}

Пусть $G=L_{2}(q)$, где $q=2^{n}$. Ввиду теоремы 3 будем считать, что $n>3$.

Докажем, что $(1) \Rightarrow(2)$. Допустим, что это неверно.

Предположим, что $n-$ непростое число и $r-$ простой делитель числа $n$. Положим $q_{0}=2^{n / r}$. Тогда $q=q_{0}^{r}$ и $q_{0} \geq 4$. Пусть $G \simeq L_{2}(q)$, где $q=2^{n}$. Исходя из теоремы 3 можно считать, что $n>3$. По лемме 1 группа $G$ содержит максимальную подгруппу $M$, изоморфную $L_{2}\left(q_{0}\right)$, а $M$ - максимальную подгруппу $H$, изоморфную $C_{2}^{n / r} \lambda C_{q_{0}-1}$. Пусть $S$ - силовская 2-подгруппа группы $G$, содержащая $O_{2}(H)$, и $P=N_{G}(S)$. Тогда $P$ - группа Фробениуса с элементарным абелевым ядром $S$ порядка $q$ и циклическим дополнением $T$ порядка $q-1$. Поскольку $S-$ $T I$-подгруппа в $G$ (см. [8, теорема II.8.2]), имеем

$$
\left.H \leq N_{G}\left(O_{2}(H)\right) \leq N_{G}(S)\right)=P .
$$

Пусть $D-$ некоторая подгруппа в $H$, изоморфная $C_{q_{0}-1}$. Можно считать, что $D<T$. Подгруппа $T$ действует транзитивно на инволюциях из $S$, следовательно, $O_{2}(H) \neq O_{2}(H)^{t}$ для некоторого элемента $t$ из $T \backslash D$. Поэтому в подгруппе

$$
F=\left\langle H, H^{t_{1}}\right\rangle=\left\langle O_{2}(H), O_{2}(H)^{t}\right\rangle D
$$

подгруппа $H$ нормальна. Но тогда подгруппы $H$ и $H^{t}$ не сопряжены в $F$. Следовательно, подгруппа $H$ не пронормальна в $G$, что противоречит условию (1). 
Итак, $n=p$ - простое число, большее 3 , и $2^{p}-1-$ простое число Мерсенна. По лемме 1 группа $G$ содержит максимальную подгруппу $M$, изоморфную $D_{2\left(2^{p}-1\right)}$, а $M$ - максимальную подгруппу $H$ порядка 2 . Пусть $S$ - силовская 2-подгруппа группы $G$, содержащая $H$, и $P=$ $N_{G}(S)$. Так как $P$ действует транзитивно на инволюциях из $\left.S\right)$, то $H \neq H^{t}$ для некоторого элемента $t$ из $P$. Поэтому $H$ и $H^{t}$ не сопряжены в подгруппе

$$
F=\left\langle H, H^{t}\right\rangle=H \times H^{t} .
$$

Следовательно, подгруппа $H$ не пронормальна в $G$, что противоречит условию (1).

Таким образом, $(1) \Rightarrow(2)$.

Докажем, что $(2) \Rightarrow(1)$. Допустим, что это неверно. Тогда $q-1-$ непростое число и группа $G$ содержит непронормальную вторую максимальную подгруппу $H$. Применим предложение в его обозначениях. Имеем $H<\cdot M<\cdot G$ и $H<F \leq M_{1}<\cdot G$ для некоторых максимальных подгрупп $M$ и $M_{1}$ из $G$. По лемме 1 каждая из подгрупп $M$ и $M_{1}$ изоморфна $C_{2}^{n} \lambda C_{(q-1)}$ или $D_{2(q \pm 1)}$. Рассмотрим эти случаи.

(a) $M \simeq C_{2}^{n} \lambda C_{(q-1)}$. Тогда максимальная в $M$ подгруппа $H$ изоморфна $C_{(q-1)}$ или $C_{2}^{n} \lambda C_{d}$, где $d$ - делитель числа $q-1$ такой, что $(q-1) / d-$ простое число.

Допустим, что $H \simeq C_{(q-1)}$. Тогда $H-$ холлова подгруппа в $G$, поэтому подгруппы $H$ и $H^{g}$ сопряжены в $F$ в силу разрешимости подгруппы $M_{1}$. Это противоречит утверждению (1) предложения.

Таким образом, $H \simeq C_{2}^{n} \lambda C_{d}$. Ясно, что $M_{1} \simeq C_{2}^{n} \lambda C_{(q-1)}$. Следовательно, $F=M_{1}$. Но $C_{d}$ - циклическая подгруппа простого индекса в $C_{q-1}$. Следовательно, $C_{d}$ - единственная подгруппа порядка $d$ в $C_{(q-1)}$. Поэтому из сопряженности холловых $2^{\prime}$-подгрупп в разрешимой группе $F$ следует сопряженность в $F$ подгрупп порядка $d$ из $F$, значит, подгруппы $H$ и $H^{g}$ сопряжены в $F$, что противоречит утверждению (1) предложения.

(б) $M \simeq D_{2(q \pm 1)}$. Тогда максимальная в $M$ подгруппа $H$ либо изоморфна $C_{(q \pm 1)}$, либо имеет четный порядок и простой индекс в $M$.

Если $H \simeq C_{(q \pm 1)}$, то $H$ - холлова подгруппа в $G$, поэтому подгруппы $H$ и $H^{g}$ сопряжены в $F$ в силу разрешимости подгруппы $M_{1}$. Это противоречит утверждению (1) предложения.

Таким образом, подгруппа $H$ имеет четный порядок и простой индекс в $M$. Поскольку $q-1-$ непростое число, подгруппа $H$ изоморфна диэдральной группе. Соответственно

$$
M_{1} \simeq D_{2(q \pm 1)} \simeq M .
$$

Следовательно, $F=M_{1}$ и $|F|_{2}=2$. Тогда для инволюции $i$ из $H$ имеем $i^{g}=i^{f}$ для некоторого элемента $f$ из $O(F)$. Так как подгруппа $O(H)$ характеристична в $O(F)$, отсюда получаем, что $H^{g}=H^{f}$. Это противоречит утверждению (1) предложения.

Таким образом, $(2) \Rightarrow(1)$.

Теорема 1 доказана.

\section{4. Доказательство теоремы 2}

Пусть $G=L_{2}(q)$, где $q=p^{n}>11, p$ - нечетное простое число и $n$ - натуральное число.

Докажем, что (1) $\Rightarrow(2)$.

Прежде всего докажем, что $q=p^{n}-$ простое число. Допустим, что это неверно. Тогда $n$ делится на некоторое простое число $r$. Поэтому $q=q_{0}^{r}$, где $q_{0}=p^{n / r}$. По п. (г) леммы 2 группа $G$ содержит максимальную подгруппу $M$, изоморфную $P G L_{2}\left(q_{0}\right)$, а по п. (а) леммы 3 $M$ содержит максимальную подгруппу $H$, изоморфную $C_{p}^{n / r} \lambda C_{q_{0}-1}$. Пусть $S-$ силовская $p$-подгруппа группы $G$, содержащая $O_{p}(H)$, и $P=N_{G}(S)$. Тогда $P$ - группа Фробениуса с элементарным абелевым ядром $S$ порядка $q$ и циклическим дополнением $T$ порядка $(q-1) / 2$. Поскольку $S-T I$-подгруппа в $G$ (см. [8, теорема II.8.2]), имеем

$$
\left.H \leq N_{G}\left(O_{p}(H)\right) \leq N_{G}(S)\right)=P .
$$


Пусть $D$ - некоторая подгруппа в $H$, изоморфная $C_{q_{0}-1}$. Можно считать, что $D<T$. Подгруппа $T$ действует транзитивно на подгруппах порядка $p$ из $S$, следовательно, $O_{p}(H) \neq O_{p}(H)^{t}$ для некоторого элемента $t$ из $T \backslash D$. Поэтому в подгруппе

$$
F=\left\langle H, H^{t}\right\rangle=\left\langle O_{p}(H), O_{p}(H)^{t}\right\rangle D
$$

подгруппа $H$ нормальна. Но тогда подгруппы $H$ и $H^{t}$ не сопряжены в $F$. Следовательно, подгруппа $H$ не пронормальна в $G$, что противоречит условию (1). Итак, $q$-простое число.

Далее докажем, что числа $\frac{q-1}{2}$ и $\frac{q+1}{2}$ не являются простыми. Предположим противное. Тогда число $(q-\epsilon) / 2$ для некоторого $\epsilon= \pm 1$ является простым. Поскольку $q>11$, по лемме 2 группа $G$ содержит максимальную подгруппу $M$, изоморфную $D_{q-\epsilon}$, причем $|M|_{2}=2$. Значит, подгруппа $I$ второго порядка из $M$ есть вторая максимальная подгруппа в $G$. Ввиду леммы 2 группа $G$ содержит подгруппу $R$, изоморфную $A_{4}$ при $q \equiv \pm 3(\bmod 8)$ и $S_{4}$ при $q \equiv \pm 1(\bmod 8)$. Поскольку инволюции в группе $G$ сопряжены, можно считать, что $I \leq O_{2}(R)$. Поэтому $I \neq I^{t}$ для некоторого элемента $t$ из $R$, причем $\left\langle I, I^{t}\right\rangle \simeq C_{2}^{2}$. Значит, подгруппа $I$ не пронормальна в $G$, что противоречит условию (1). Итак, числа $\frac{q-1}{2}$ и $\frac{q+1}{2}$ не являются простыми.

Докажем теперь, что $\left(q^{2}-1\right)_{2} \leq 2^{5}$. Предположим противное. Тогда $q \equiv \pm 1(\bmod 8)$ и, следовательно, по п. (и) леммы 2 группа $G$ содержит максимальную подгруппу $M$, изоморфную $S_{4}$. Группа $M$ содержит максимальную подгруппу $D$, изоморфную $D_{8}$. Пусть $S-$ силовская 2-подгруппа группы $G$, содержащая $D$. Из неравенства $\left(q^{2}-1\right)_{2}>2^{5}$ следует, что $|S|>2^{4}$. Так как подгруппа $S$ диэдральна, то $N_{S}(D) \simeq D_{16}$. Следовательно, $D \neq D^{t}$ для некоторого элемента $t$ из $N_{S}\left(N_{S}(D)\right) \backslash N_{S}(D)$. Тогда подгруппа $F=\left\langle D, D^{t}\right\rangle$ равна $N_{S}(D)$. Поскольку $|F: D|=2$, подгруппа $D$ нормальна в $F$. Поэтому $D$ не пронормальна в $G$, что противоречит условию (1). Итак, $\left(q^{2}-1\right)_{2} \leq 2^{5}$.

Осталось доказать, что если $q \equiv \varepsilon(\bmod 8)$, где $\varepsilon= \pm 1$, то

$$
q \equiv-\varepsilon(\bmod 3) \text { и } q \equiv-\varepsilon(\bmod 15) \text { при } q \equiv \pm 1(\bmod 10) .
$$

Допустим, что $q \equiv \varepsilon(\bmod 8)$, где $\varepsilon= \pm 1$, т. е. $8 \mid(q-\varepsilon)$. По п. (б) леммы 2 группа $G$ содержит максимальную подгруппу $M$, изоморфную $D_{q-\varepsilon}$. Если $O(M)=1$, то условия $q>11$ и $\left(q^{2}-1\right)_{2} \leq 2^{5}$ влекут, что $q=17$ и доказываемое утверждение справедливо. Значит можно считать, что $O(M) \neq 1$.

Покажем, что диэдральные подгруппы двойного нечетного порядка из $M$ не пронормальны $M$, а значит, и в $G$. Действительно, пусть $H$ - такая подгруппа и $I$ - подгруппа порядка 2 в $H$. Так как $8 \mid(q-\varepsilon)$, подгруппа $I O_{2}(M)$ является неабелевой диэдральной 2-группой и, следовательно, $I \neq I^{t}$ для некоторого элемента $t$ порядка 4 из $O_{2}(M)$. Подгруппа $O(H)$ централизует $\mathrm{O}_{2}(M)$, поэтому в подгруппе $F=\left\langle H, H^{t}\right\rangle=O(H)\left(I \times I^{t}\right)$ подгруппа $H$ нормальна. Но тогда подгруппы $H$ и $H^{t}$ не сопряжены в $F$. Таким образом, подгруппа $H$ не пронормальна в $M$.

По лемме 2 группа $G$ содержит максимальную подгруппу $M$, изоморфную $S_{4}$. Группа $M$ содержит максимальную подгруппу $H$, изоморфную $S_{3}$. Как показано в предыдущем абзаце, в силу условия (1) подгруппа $H$ не может лежать в максимальной подгруппе из $G$, изоморфной $D_{q-\varepsilon}$. Поэтому ввиду леммы 2 подгруппа $H$ содержится в максимальной подгруппе из $G$, изоморфной $D_{q+\varepsilon}$. Следовательно, $3 \mid(q+\varepsilon)$, т. е. $q \equiv-\varepsilon(\bmod 3)$.

Предположим, что $q \equiv \pm 1(\bmod 10)$. Тогда по лемме 2 группа $G$ имеет максимальную подгруппу $M_{1}$, изоморфную $A_{5}$, а группа $M_{1}$ содержит максимальную подгруппу $H$, изоморфную $D_{10}$. Рассуждая как в предыдущем абзаце, получим, что подгруппа $H$ содержится в максимальной подгруппе из $G$, изоморфной $D_{q+\varepsilon}$. Следовательно, $15 \mid(q+\varepsilon)$, т. е. $q \equiv-\varepsilon(\bmod 15)$.

Таким образом, $(1) \Rightarrow(2)$.

Докажем, что $(2) \Rightarrow(1)$. Допустим, что это неверно и выполнено условие (2). Тогда группа $G$ содержит непронормальную вторую максимальную подгруппу $H$. Применим предложение в его обозначениях. Имеем $H<\cdot M<\cdot G$ и $H<F \leq M_{1}<\cdot G$ для некоторых максимальных 
подгрупп $M$ и $M_{1}$ из $G$. По лемме 2 каждая из подгрупп $M$ и $M_{1}$ изоморфна одной из следующих групп: $C_{q} \lambda C_{(q-1) / 2}, D_{q \pm 1)}, A_{5}$ при $q \equiv \pm 1(\bmod 10), A_{4}$ при $q=p \equiv \pm 3(\bmod 8)$ и $q \not \equiv \pm 1(\bmod 10)$ или $S_{4}$ при $q=p \equiv \pm 1(\bmod 8)$. Рассмотрим эти случаи. Заметим, что $(q-1) / 2 \geq 6$ ввиду неравенства $q \geq 13$ и числа $(q-1) / 2$ и $(q+1) / 2$ взаимно просты.

Предположим, что $M \simeq C_{q} \lambda C_{(q-1) / 2}$. Тогда либо $q$ делит $|H|$, либо $H \simeq C_{(q-1) / 2}$.

Пусть $q$ делит $|H|$. Тогда $M_{1} \simeq M$ и, следовательно, $H<F=M_{1}$. Поскольку подгруппа порядка $|H|$ в $F$ единственна, имеем $H^{g}=H$, что противоречит утверждению (1) предложения. Поэтому $H \simeq C_{(q-1) / 2}$. Если $M_{1} \simeq M$, то $H<F=M_{1}$ и, значит, циклические подгруппы порядка $(q-1) / 2$ сопряжены в $F$, что противоречит утверждению (1) предложения. Поэтому $M_{1} \simeq D_{q-1}$ и, следовательно, $H<F=M_{1}$. Поскольку циклическая подгруппа порядка $|H|$ в $F$ единственна, имеем $H^{g}=H$, что противоречит утверждению (1) предложения.

Предположим, что $M \simeq D_{q-\varepsilon}$, где $\varepsilon= \pm 1$. По теореме Хупперта [8, теорема VI.9.2] индекс $|M: H|=r-$ простое число.

Если $M_{1} \simeq C_{q} \lambda C_{(q-1) / 2}$, то $H \simeq C_{(q-1) / 2}$ и, значит, $H \cdot F=M_{1}$. Но тогда все подгруппы порядка $(q-1) / 2$ сопряжены в $F$, что противоречит утверждению (1) предложения.

Пусть $M_{1} \simeq D_{q \pm 1}$. Тогда $M_{1} \simeq D_{q-\varepsilon}$ и, значит, $H \cdot F=M_{1}$. В $M_{1}$ имеется единственная циклическая подгруппа $Z$ индекса 2 . Ввиду утверждения (1) предложения подгруппа $H$ не содержится в $Z$ и, таким образом,

$$
|Z: H \cap Z|=r, \quad H \cap Z \triangleleft M_{1} \text { и } \quad M_{1} / H \cap Z \simeq D_{2 r} .
$$

Если $r>2$, то подгруппы $H$ и $H^{g}$ сопряжены в $F$, что противоречит утверждению (1) предложения. Поэтому $r=2$ и, следовательно, $H \triangleleft M_{1}$. Если $O(H) \neq 1$, то $O\left(M_{1}\right)=O(H)=$ $O\left(H^{g}\right)=O(H)^{g}$ и поэтому $g \in N_{G}\left(O\left(M_{1}\right)\right)=M_{1}$, откуда $H=H^{g}$, что противоречит утверждению (1) предложения. Таким образом, $O\left(M_{1}\right)=1$. Поскольку $q-\varepsilon \geq 10$ и $\left(q^{2}-1\right)_{2} \leq 2^{5}$, получаем $q-\varepsilon=16$, т. е. $\varepsilon=1, q=17$ и $H \simeq D_{8}$. В $M_{1}$ имеется единственная циклическая подгруппа $L$ порядка 4 , соответственно $g \in N_{G}(L)=M_{1}$, откуда $H=H^{g}$, что противоречит утверждению (1) предложения.

Предположим, что $M \simeq A_{4}$ при $q=p \equiv \pm 3(\bmod 8)$. Тогда $H$ изоморфна $2^{2}$ или $C_{3}$. Если $H \simeq 2^{2}$, то $H \simeq \operatorname{Syl}_{2}(G)$ и, исходя из этого, $H$ и $H^{g}$ сопряжены в $F$, что противоречит утверждению (1) предложения. Поэтому $H \simeq C_{3}$ и, следовательно, 3 делит $q-\varepsilon$ для некоторого $\varepsilon= \pm 1$. По лемме 2 силовские 3 -подгруппы в $G$ циклические. Исходя из этого подгруппы порядка 3 в $F$ сопряжены, что противоречит утверждению (1) предложения.

Предположим, что $M \simeq A_{5}$ при $q=p \equiv \pm 1(\bmod 10)$. Тогда $H$ изоморфна $A_{4}, D_{6}$ или $D_{10}$. Если $H \simeq A_{4}$, то $M_{1}$ изоморфна $A_{5}$ или $S_{4}$ и, следовательно, $H \cdot F=M_{1}$. Но подгруппы, изоморфные $A_{4}$, в $F$ сопряжены, что противоречит утверждению (1) предложения.

Таким образом, $H \simeq D_{2 r}$ для $r \in\{3,5\}$. Если $M_{1}$ изоморфна $A_{5}$ или $S_{4}$, то приходим к противоречию, как в предыдущем абзаце. Соответственно $M_{1} \simeq D_{q-\varepsilon}$ для некоторого $\varepsilon= \pm 1$. По лемме 2 силовские $r$-подгруппы в $G$ циклические. Поэтому подгруппы порядка $r$ в $F$ сопряжены, в частности, существует элемент $f \in F$ такой, что $O_{r}(H)^{g}=O_{r}(H)^{f}$. Отсюда $N_{G}\left(O_{r}(H)\right)^{g}=N_{G}\left(O_{r}(H)\right)^{f}$. Но $Z:=N_{G}\left(O_{r}(H)\right)$ - единственная циклическая подгруппа индекса 2 в $M_{1}$. Исходя из этого $Z^{g}=Z^{f}=Z$ и, следовательно, $g \in M_{1}$. Поскольку $M_{1}=Z H$, можно считать, что $g \in Z$. Если 8 делит $q-\varepsilon$, то по условие $(2)$ влечет, что $r$ делит $q+\varepsilon$; противоречие. Поэтому $(q-\varepsilon)_{2} \leq 4$ и, следовательно, $O_{2}\left(M_{1}\right)=O_{2}(Z)=Z\left(M_{1}\right)$ и $\left|Z\left(M_{1}\right)\right| \leq 2$. Так как $H^{g} \neq H$, можно считать, что $1 \neq g \in O(Z)$. Но тогда инволюция $i$ из $H$ инвертирует элемент $g$ и, следовательно, $g \in\left\langle i, i H^{g}\right\rangle \leq F$, что противоречит утверждению (1) предложения.

Предположим наконец, что $M \simeq S_{5}$ при $q \equiv \pm 1(\bmod 8)$. Тогда $H$ изоморфна $A_{4}$ или $D_{6}$, и мы приходим к противоречию, как в предыдущем абзаце.

Таким образом, $(2) \Rightarrow(1)$.

Теорема 2 доказана. 


\section{СПИСОК ЛИТЕРАТУРЫ}

1. Legovini P. Gruppi finiti i cui sottogruppi sono o subnormali o pronormali // Rend. Sem. Math. Univ. Padova. 1981. Vol. 65. P. 47-51.

2. Ли С., Чжан С. Об индексах подгрупп в соединении их сопряженных пар // Сиб. мат. журн. 2013. T. 54, № 4. C. 826-837.

3. Vdovin E.P., Revin D.O. Pronormality of Hall subgroups in finite simple groups // Siberian Math. J. 2012. Vol. 53, no 3. C. 419-430.

4. Kondrat'ev A.S., Maslova N.V., Revin D.O. On the pronormality of subgroups of odd index in finite simple groups // Siberian Math. J. 2015. Vol. 56, no. 6. P. 1101-1107.

5. Kondrat'ev A.S., Maslova N.V., Revin D.O. A pronormality criterion for supplements to abelian normal subgroups // Proc. Steklov Inst. Math. (Suppl.) 2017. Vol. 296, Suppl. 1. P. 145-150.

6. Unsolved problems in group theory. The Kourovka notebook. No. 19 / eds. Evgeny Khukhro and Victor Mazurov [e-resource]. Novosibirsk, 2018 (2020). 250 p. URL: http://kourovka-notebook.org .

7. Dickson L.E. Linear Group: with an exposition of the Galois field theory. Teubner: Leipzig, 1901 (N Y: Dover Publ., Inc. 1958).

8. Huppert B. Endliche Gruppen I. Berlin; Heidefferg; N Y: Springer-Verlag, 1967.793 p.

9. Giudici M. Maximal subgroups of almost simple groups with socle $P S L(2, q)$ [e-resource]. 2007. 11 p. URL: http://arxiv.org/abs/math/0703685 .

10. Bray J.N. [at al.] The maximal subgroups of the low-dimensional finite classical groups. Cambridge: University Press, 2013. 435 p. doi: 10.1017/CBO9781139192576.

11. Conway J.H. [et. al.] Atlas of finite group. Oxford: Clarendon Press, 1985. 252 p.

12. Aschbacher M. Finite group theory. Cambridge: Cambridge University Press, 1986. 274 p.

Поступила 29.10.2019

После доработки 11.07.2020

Принята к публикации 3.08.2020

Зенков Виктор Иванович

д-р физ.-мат. наук, ведущий науч. сотрудник

Институт математики и механики им. Н.Н. Красовского УрО РАН;

Уральский федеральный университет

г. Екатеринбург

e-mail: v1i9z52@mail.ru

\section{REFERENCES}

1. Legovini P. Gruppi finiti i cui sottogruppi sono o subnormali o pronormali. Rend. Sem. Math. Univ. Padova, 1981, vol. 65, pp. 47-51.

2. Li X., Zhang X. On indices of subgroups in the join of their conjugate pairs. Siberian Math. J., 2013, vol. 54, no. 4, pp. 656-665. doi: 10.1134/S0037446613040071.

3. Vdovin E.P., Revin D.O. Pronormality of Hall subgroups in finite simple groups. Siberian Math. J., 2012, vol. 53, no 3, pp. 419-430. doi: 10.1134/S0037446612020231.

4. Kondrat'ev A.S., Maslova N.V., Revin D.O. On the pronormality of subgroups of odd index in finite simple groups. Siberian Math. J., 2015, vol. 56, no. 6, pp. 1101-1107. doi: 10.1134/S0037446615060142 .

5. Kondrat'ev A.S., Maslova N.V., Revin D.O. A pronormality criterion for supplements to abelian normal subgroups. Proc. Steklov Inst. Math., 2017, vol. 296, suppl. 1, pp. 145-150. doi: $10.1134 /$ S0081543817020134.

6. Unsolved problems in group theory. The Kourovka notebook [e-resource], No. 19, eds. Evgeny Khukhro and Victor Mazurov, Novosibirsk, 2018 (2020), 250 p. Available at: http://kourovka-notebook.org .

7. Dickson L.E. Linear Group: with an exposition of the Galois field theory. Teubner: Leipzig, 1901. (N Y: Dover Publ., Inc. 1958).

8. Huppert B.Endliche Gruppen I. Berlin; Heidefferg; N Y: Springer-Verlag, 1967. 793 p.

9. Giudici M. Maximal subgroups of almost simple groups with socle $\operatorname{PSL}(2, q) .2007 .11$ p. Available on https://arxiv.org/abs/math/0703685.

10. Bray J.N. et al. The maximal subgroups of the low-dimensional finite classical groups. Cambridge: University Press, 2013, 435 p. doi: 10.1017/CBO9781139192576. 
11. Convay J.H. et al. Atlas of finite group. Oxford: Clarendon Press, 1985, 252 p. ISBN: 0-19-853199-0 . 12. Aschbacher M. Finite group theory. Cambridge: Cambridge University Press, 1986. 274 p.

Received October 29, 2019

Revised July 11, 2020

Accepted August 3, 2020

Funding Agency: This work was supported by the Russian Academic Excellence Project (agreement no. 02.A03.21.0006 of August 27, 2013, between the Ministry of Education and Science of the Russian Federation and Ural Federal University).

Victor Ivanovich Zenkov, Dr. Phys.-Math. Sci., Krasovskii Institute of Mathematics and Mechanics Ural Branch of the Russian Academy of Sciences, Yekaterinburg, 620108, Russia; Ural Federal University, Yekaterinburg, 620083 Russia, e-mail: v1i9z52@mail.ru .

V. I. Zenkov. On pronormality of second maximal subgroups in finite groups with socle $L_{2}(q)$, Trudy Instituta Matematiki i Mekhaniki URO RAN, 2020, vol. 26, no. 3, pp. 32-43. 